\section{Großer versus kleiner Umsatzkreis}

\author{
Welche psychologischen Grundlagen liegen unseren Konsumentscheidungen \\ zugrunde? Der Normalkonsum lässt sich durch sechs psychologische Kennzei- \\ chen charakterisieren, die die Eckpunkte eines Spannungsgefüges bilden. Wie \\ eine tiefenpsychologische Pilotstudie über das zeitgenössische Konsumverhal- \\ ten in Deutschland zeigt, unterscheiden sich die seelischen Grundfaktoren des \\ Öko-Konsums ganz erheblich davon. Das Bewusstmachen seines kleineren \\ „Umsatzkreises" kann zum Beispiel helfen, die Marktchancen von Öko-Kauf- \\ häusern einzuschätzen (1).
}

$\mathrm{D}$

Von Wolfram Domke er sogenannte Normalkonsum lässt sich durch sechs psychologische Kennzeichen beschreiben. Aus Darstellungsgründen werden diese Kennzeichen einzeln und nacheinander vorgestellt, was vielleicht den Eindruck erwecken könnte, es handele sich hier um unabhängige Faktoren. Tatsächlich markieren die Kennzeichen jedoch Eckpunkte eines in sich zusammenhängenden Spannungsgefüges oder Gliedzüge einer Wirkungseinheit (siehe Abbildung 1 auf S. 17). Zusammen bilden die psychologischen Strukturmerkmale den großen Umsatzkreis, der den Normalkonsum kennzeichnet.

\section{- Lebenshungrige Eigenwelt}

Die psychologische Ausgangslage des Normalkonsums ist ein Seelenzustand, der in gewisser Weise dem eines Kleinkindes ähnelt: Das Eigene wird als etwas prinzipiell Unselbständiges, Unfertiges erlebt, das zum Überleben dringend auf ständige Zufuhr von außen angewiesen ist. Ein Grundgefühl ständigen Hungers und nie endender Bedürftigkeit motiviert das fortwährende Kaufen von Waren. Mit ihrem Konsum verbinden sich die wichtigen psychologischen Qualitäten von Konstanz, Sicherheit und Halt. Die große Bedeutung dieser Versorgungssicherheit lässt sich am anschaulichsten in Notzeiten beobachten: Auf die Verknappung wird mit panischen Hamsterkäufen reagiert. In Wohlstandszeiten kommt das ständige Zufuhrbedürfnis in anderer Weise zum Ausdruck: etwa in den verschiedenen Suchtformen des Essens, Trinkens oder des Kaufens selber.

\section{- Direkter Weltzugang}

Ein zweites Kennzeichen des Normalkonsums zeigt sich in der ausgeprägten Tendenz zur schnellen Bedürfnisbefriedigung. Das hungrige Grundgefühl schafft bei den Verbrauchern eine latente Offenheit für die verschiedensten Produkte. Sobald diese Offenheit durch nahegelegte Angebote spürbar wird, drängt sie auf sofortige Schließung, also auf einen Kauf. Verallgemeinert heißt das: Die Warenwelt erscheint im Normalkonsum wie ein Endloslaufband voller Objekte der Begierde, die man am liebsten hier und jetzt besitzen oder sich direkt einverleiben möchte. Auch das erinnert an die Unduldsamkeit der frühkindlichen Aneignung. Die Entfaltung einer lustvollen Augenblickslogik macht stärker empfänglich für die sinnlichen Reize der Welt und ermöglicht damit auch ein besonderes Genusserlebnis im Konsum.

\section{Reiche Welt als Ideal}

Während der Zug der Direktheit den Umsatzkreis des Normalkonsums beschleunigt, erfährt er durch sein immanentes Reichtumsideal eine deutliche Ausdehnung. Was man auch hat, nie scheint ein Ende der Begehrlichkeiten, eine Grenze des Ersehnten in Sicht zu kommen. Stets richten sich die Konsumträume und -sehnsüchte auf ein allzeit verlockendes Darüberhinaus und Mehr. Besonders in gesteigerten Konsumausgaben wird hier spürbar, dass man sich aus kleinen und beengten Verhältnissen herausgearbeitet hat. Ein reicher Umsatzkreis stellt damit einen sichtbaren Maßstab für das Höher- und Weiterkommen der eigenen Entwicklung dar. Daraus erklärt sich auch die Anziehungskraft der schönen Reklamebilder: Sie werben mit für das Reichtumsideal und bieten ihm immer weitere Etappenziele.

\section{Drängen auf Neu-Umsatz}

In der Psycho-Logik des Normalkonsums spielt das Neue eine überaus wichtige Rolle. Neu ist grundsätzlich all das, was im strukturellen Gegenpol der Eigenwelt nicht enthalten ist, was zunächst einmal unvertraut, ungewohnt und fremd erscheint. Diesem Fremden steht der Normalkonsum besonders aufnahmefreudig gegenüber: Gerne ist man hier bereit, seine alten Bestände gegen neue Errungenschaften auszutauschen. Ohne einen solchen Austausch erlebt man seine eigene Entwicklung als stagnierend oder gar rückläufig. Umgekehrt verspricht man sich von der Steigerung seines Warenumsatzes eine Belebung und Erneuerung des eigenen Lebens.

Dieser Strukturzug macht die psychologische Attraktivität von Moden verständlich: Ein neues Gewand vermag die wiederkehrende Aneignung des grundsätzlich Gleichen (Lebensmittel, Kleider, Möbel) so schmackhaft zu machen, dass es zum zyklischen Wechsel zwischen Ausgedientem und Unverbrauchtem kommt. Insgesamt ist alles Neue deshalb ein zugkräftiges Verkaufsargument für die Verbraucher, weil das Seelische selbst neugierig ist, also auf Veränderung drängt.

\section{Zwang zum Maßhalten}

Selbst die konsumfreudigsten Kunden besitzen bestimmte Limits, um deren strenge Einhaltung sie sich im Konsum zumindest bemühen. Einschätzungen wie ,zu viel“ und ,zu teuer“ markieren solche Grenzen, an denen eine Überschreitung der eigenen Verhältnisse deutlich verspürt wird. Auch wenn sich dieses Überschreitungsgefühl auf sehr verschiedenen Preisniveaus einstellen kann, so weist es doch generell auf das Vorhandensein eines wirksamen Konsummaßes hin. Zur PsychoLogik dieses Strukturzuges gehört auch eine ausgesprochen realistische Weltsicht. Realistisch heißt hier: Verschärfung des Blickes für das Lebensnotwendige, besondere Beachtung des Machbaren und Möglichen, kritische Abweisung des Unnötigen und Luxuriösen.

Eine solch sparsame Konsumhaltung wird unter dem Druck beschränkter finanzieller Mittel zwar gefördert, aber sie zeigt sich durchaus auch in reichen Verhältnissen, etwa als Geiz. Der Zwang zum Maßhalten wirkt im Gesamtzusammenhang des Normalkonsums wie eine ökonomische Bremse, welche die Aufrechterhaltung eines bestimmten Grundumsatzes sicherstellt.

\section{Gewinn-und-Verlust-Denken}

„Geld regiert die Welt" - das ist die Verrechnungsdevise des Normalkonsums: Wer viel Geld hat, kann sich viel von den Waren der fremden Welt aneignen; wer wenig hat, kann sich nur weniger Austausch leisten. Geld wird damit zum entscheidenden Maßstab für die Verteilungsgerechtigkeit 
zwischen eigener und fremder Welt. Das ist ein egozentrischer Maßstab, weil die Gewinn und Verlustrechnung des Konsums stets eine sehr persönliche ist: Immer steht der eigene Vorteil, aber auch der eigene Schaden im Vordergrund des Konsumerlebens. Glück und Unglück der eigenen Entwicklung erscheinen innig verbunden mit dem Erreichen eines möglichst großen, reichbewegten Umsatzkreises, der im Ganzen profitabel sein soll. Profitabel heißt hier: mehr entnehmen als zurückgeben. Das Gelingen eines solchen ökonomischen Kunststïckes führt - wie etwa bei einem Schnäppchenkauf - zu einem ,,seelischen Händereiben“; schlechtes Gewissen bereitet eher sein Misslingen.

\section{- Anders ausgeprägt: der kleine Umsatzkreis des Öko-Konsums}

Nachfolgend werden nun - in struktureller Analogie zur psychologischen Analyse des Normalkonsums als großem Umsatzkreis - sechs Kennzeichen des Öko-Konsums beschrieben. Dabei zeigt sich, dass dieselben seelischen Grundfaktoren hier in einer deutlich anderen Ausprägung erscheinen. Dieser Andersartigkeit trägt die Benennung als kleiner Umsatzkreis ganzheitlich Rechnung.

\section{- Autarke „Ei-Welt"}

Ein banal scheinende Änderung der alltäglichen Essgewohnheiten steht oft am Anfang des ÖkoKonsums: die angewiderte Abkehr von den ,nach Fischmehl schmeckenden Legebatterieprodukten“ und die bewusste Hinwendung zum „natürlich schmeckenden Bio-Ei““. Daneben wurde eine weitere, signifikante Startstelle für den Öko-Konsum auffällig: Das Kinderkriegen. Den eigenen Kindern und ihrer Gesundheit zuliebe beginnt eine zum Teil gravierende Umstellung des bisherigen Konsumverhaltens. Zwischen Ei und Kindern besteht eine psychologische Korrespondenz: beide symbolisieren hier einen vielversprechenden Neuanfang für das Eigene. Geschützt vor schädlichen Einwirkungen der Umwelt soll die Entwicklung wieder ganz natürlich in Gang kommen und - soweit dies in Gesellschaft anderer überhaupt möglich ist - auch in sich bleiben.

Erst vor diesem Ei-Bild wird deshalb verständlich, warum sich im Öko-Konsum ein solch machtvoller Drang zum Rückzug und zur Abkapselung zeigt: Tendenziell geht es hier um den Aufbau einer möglichst autarken Familieneinheit, die sich weitgehend selbst versorgt und gleichsam aus sich selbst heraus wächst und gedeiht.

\section{Biologisch-kontrollierter Weltzugang}

Wie manche alternativen Lebensexperimente zeigen, führt eine extreme Abschottung der Eigenwelt bald an existenzgefährdende Grenzen heran. Als genauso lebensbedrohlich wird es hier aber auch erlebt, wenn der Außenwelt in naiver Bedenkenlosigkeit gewissermaßen Tür und Tor geöffnet wird. Im Öko-Konsum bemüht man sich daher um eine Kompromissform: Der Zugang zur Außenwelt wird nicht radikal versperrt, aber die Öffnung geschieht nur stark gefiltert. Es entwickelt sich ein psychologisches Filtersystem, das seine abwehrende Wirkung bereits in der Wahrnehmung entfaltet: Alles,

\section{Abb. 1: Umsatzkreise des Normalkonsums (außen) und des Ökokonsums (innen)}

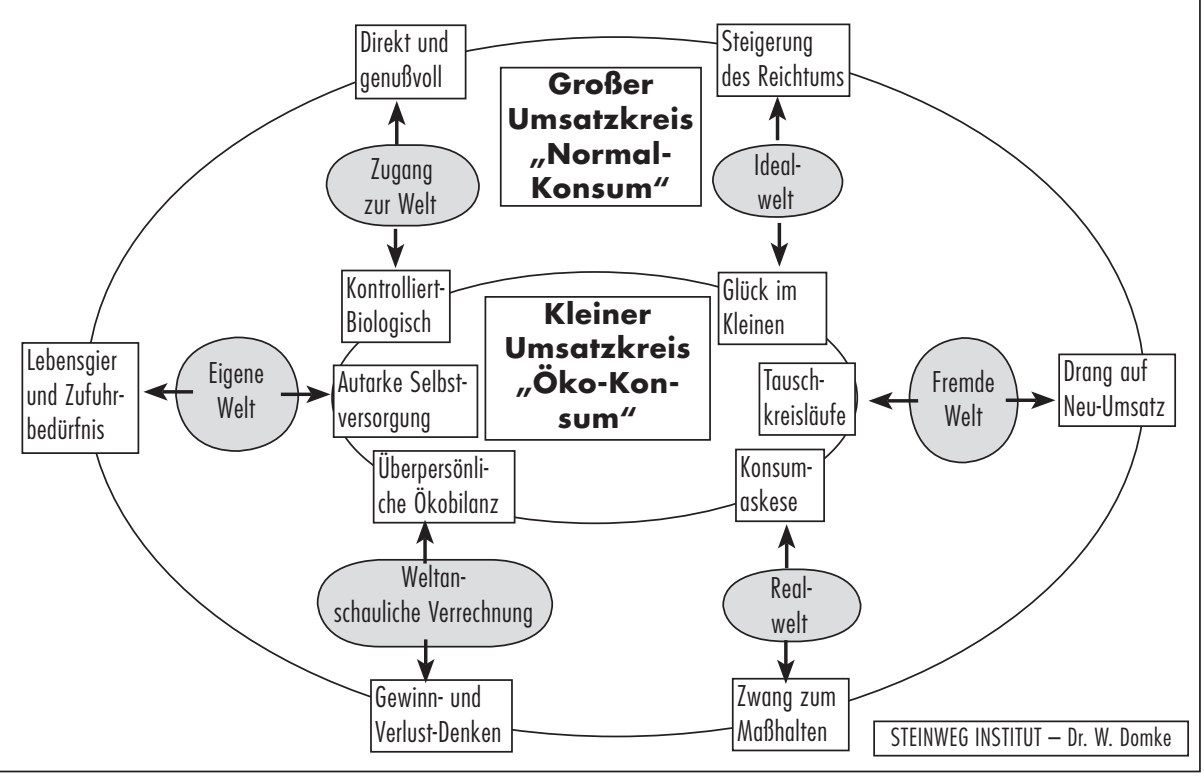

was die Sinne durch leuchtende Farben, verlokkende Düfte und Geschmäcker zum spontanen Genuss verführen könnte, wird misstrauisch beäugt und tendenziell als gefährlich oder gar schädlich eingestuft. Fast schon so, als ginge es im alltäglichen Konsum wieder um die teuflische Versuchung durch den paradiesischen Apfel, zwingt man sich vor allem bei lustvollen Sinneserregungen zu standhaftem Verzicht. Zum Konsum zugelassen werden nur Waren, denen nach aufwendiger Überprüfung eine ökologische Unbedenklichkeit bescheinigt werden kann. Einschlägige Öko-Zeitschriften werden dabei zur Unterstïtzung und Beglaubigung der eigenen Kontrollarbeit gerne genutzt.

\section{Idealwelt: Glück im Kleinen}

Auch im Öko-Konsum gibt sich ein übergreifendes Ideal zu erkennen, das etwas Unerreichbares besitzt, und dennoch eine stark motivierende Wirkung auf das alltägliche Handeln ausübt. Anders als im Normalkonsum ist dieses Ideal nicht auf das progressive Erreichen materiellen Reichtums gerichtet. Vielmehr gibt es sich in dem geradezu bescheiden anmutenden Bild zu erkennen, das etwa Voltaire in seinem Candide als schlussendliche Glücksempfehlung entwirft: „Den eigenen Garten bestellen“.

Erstaunlicherweise trifft dieses alte Idealbild auch auf die zeitgenössische Öko-Bewegung zu - und zwar in mehreren Hinsichten. Zunächst einmal ist es ein ausdrücklich ,zurückgenommenes“ Lebensideal: Wie der Romanheld Candide muss man sein Glück zunächst in der großen Welt gesucht haben, um dann erst zur Einsicht zu gelangen, dass es womöglich eher im Kleinen, im Eigenen und im Natürlichen zu finden ist. Das gilt auch für den Öko-Konsum: Man kennt sehr wohl die Vorzüge von Kultur, Zivilisation und technischem Fortschritt, doch nun verbindet man damit nicht mehr sein Entwicklungsideal. Man sucht dieses vielmehr in einem „natürlichen Wachstum“, das sich im begrenzten Kreis des Eigenen vollzieht, und darin weitgehend geschützt ist vor gefährlichen Auswüchsen.

\section{- Geschlossene Tauschkreisläufe}

Generell zeigt sich der Öko-Konsum stark darum bemüht, dem Umsatz mit fremden Waren so weit wie möglich einzuschränken - besonders, wenn diese Güter neu produziert sind und von weit her kommen. Den lebensnotwendigen Umsatz des Eigenen mit dem Fremden sucht man eher durch 
Rückgriffe auf Altbewährtes (Werkzeuge, Haushaltsgeräte), Gebrauchtes (Kinderkleidung) und Heimisches (Obst und Gemüse aus der Region) zu schaffen. Die gerne besuchten Flohmärkte illustrieren das: Das früher dem Eigenen sehr Nahestehende, aber aus der Mode Gekommene, wird als etwas Brauchbares oder gar Schönes wiederentdeckt und durch Erwerb erneut in den eigenen Umsatzkreislauf aufgenommen.

Insgesamt tendiert der Öko-Konsum eindeutig zum Aufbau möglichst geschlossener Umsatzkreise, in denen das in der Welt bereits Vorhandene am liebsten nur ausgetauscht wird. Im Idealfall entwickelt sich ein Tauschhandel wie in früheren Zeiten, bei dem Güter und Dienstleistungen direkt - also ohne Geld - hin und her wechseln. Wiederverwendung und Wiederverwertung sind hier ebenfalls Werte, welche die betont konservative Umsatzlogik des Öko-Konsums markieren. Ob Altpapier oder alte Kleider: Immer geht es darum, das bereits Bestehende zu bewahren oder durch Recycling erneut dem Umsatzkreislauf zuzuführen, damit nichts Wertvolles verlorengeht. Dass diese Öko-Kreise sich enger, einfacher, langsamer und länger drehen, dafür ist das Fahrrad fast zum Inbegriff geworden.

\section{Asketische Konsumfreuden}

Äußerlich sehr ähnlich den Sparsamkeitsbemühungen im Normalkonsum, zeigen sich auch im Öko-Konsum deutliche Reduzierungsanstrengungen - hier aber getreu der Umsatzdevise „,weniger ist mehr“. Die Paradoxie dieser Devise zieht sich wie ein roter Faden durch die PsychoLogik dieses Strukturzuges: Weniger Konsum wird erlebt als ein Mehr an Lebensqualität. Man spart Energie, schränkt seinen Fleischverbrauch ein, bekleidet seine Kinder mit Second-HandWare - solche und viele andere Reduzierungsmaßnahmen erwachsen im Öko-Konsum in der Regel nicht aus wirtschaftlichen Sparzwängen,

\section{Beilagenhinweis}

\section{Unsere Publikation enthält eine Beilage des Vablen-Verlages}

\section{Wir bitten um freundliche Beachtung.}

sondern vollziehen sich in durchaus wohlhabenden Verhältnissen. Und auch darin zeigt sich die Paradoxie dieses Zuges: Man muss schon in gewissem Sinne reich sein, um sich das vergleichsweise teure „Weniger“" im Öko-Konsum überhaupt leisten zu können.

Diese freiwillige Selbstbeschränkung mit ihrer Umwertung der normalen Lebenswerte muss psychologisch als Askeseform verstanden werden. Tatsächlich brachte die Untersuchung zutage, dass der Öko-Konsum (besonders im Lebensmittelbereich) oftmals wie eine Bußprozession für vorangegangene „Sünden“ durch ausschweifende sinnliche Genüsse betrieben wird. So werden aus unbeschwerten Konsumfreuden ernsthafte Gewissensqualen und aus leidvollen Genussentsagungen eigentümliche Selbstreinigungsfreuden.

\section{- Überpersönliche Öko-Bilanz}

Auch die unvermeidlich auftretenden Widersprüche und Konflikte im praktizierten ÖkoKonsum werden nach dem Schema von Sünde und Buße psychologisch verrechnet. Die Religionsnähe dieser Verrechnung zeigt sich auch darin, dass sich der Öko-Konsum mehr auf Seiten einer paradiesischen Unschuld stehen sieht, während er dem Normalkonsum eher die Seite teuflischer Verfübrung und menschlicher Schwäche zuweist. Aus einer solchen weltanschaulichen Aufteilung lässt sich wiederum ein schlichtes Gut-und-Böse-Schema ableiten, das für alltägliche Konsumentscheidungen sehr hilfreich ist: So sind beispielsweise Chiquita-Bananen böse und Transfair-Kaffee gut.

Eine weitere Verrechnungsart wird im Aufstellen und Beachten von Öko-Bilanzen deutlich. Auch hier zeigt sich ein schlechtes Gewissen, aber es ist psychologisch schon bemerkenswert, dass es aus der Berïcksichtigung von Kosten und Nutzen im Weltmaßstab erwächst. Im Öko-Konsum dehnt sich der Verantwortungsbereich für das Handeln weit über das persönliche Profitdenken aus in die Ökonomie des Ganzen. Dadurch erscheinen gute und böse Taten im Öko-Konsum eigentümlich überdimensioniert: „,Riesenschuldgefühle“ müssen sich hier mit einem „RiesenAltruismus" verrechnen.

\section{Schlussbemerkung}

Obwohl sie sich äußerlich oftmals feindlich gegenüberstehen, bilden traditioneller und alternativer Konsum eine strukturelle Wirkungseinheit, die in der vorliegenden Untersuchung als „Robinson-Konstruktion“ herausgearbeitet werden konnte. Aus ihr ließ sich dann eine Reihenbildung von fünf Konsumtypen ableiten, die sich vor allem zwischen den Extremen als aufschlussreich erweist. Hier zeigt sich nämlich, dass der praktizierte Konsumalltag viel mehr an einer pragmatischen Verbindung als an einer radikalen Trennung der beiden Umsatzkreise interessiert ist. Dieses Verbindungsinteresse hängt damit zusammen, dass weder der normale noch der ökologische Konsum eine ganzheitliche Lösung darstellen: Beide haben eine psychologische Moral und bieten psychologische Genüsse, doch beide lassen auch wichtige seelische Rechnungen offen. Gerade diese Offenheit fordert scheinbar dazu heraus, sich immer wieder neu und anders an der ,Quadratur des Konsumkreises" $\mathrm{zu}$ versuchen.

\section{Anmerkung}

(1) Der Beitrag beruht auf einer im Auftrag des Wuppertal Instituts (WI) vom Kölner Steinweg Institut im Sommer 1999 durchgeführten tiefenpsychologischen Pilotstudie über das zeitgenössische Konsumverhalten in Deutschland. Diese bildete einen Baustein eines Verbundprojekts, dass sich der konzeptionellen Entwicklung von Öko-Kaufhäusern widmete. Die Studie wurde in einen Gesamtforschungsbericht der Energieabteilung des WI integriert, der (bisher) unveröffentlicht ist. Weitere Beteiligte des Gesamtprojekts waren neben dem WI das IÖW (Arbeitspakete Betreiberkonzept, Finanzkonzept, Logistik, Qualifizierung), die Agentur econcept, der BUND und als Koordinator das caf clearinghouse for applied futures (vgl. Ökologisches Wirtschaften

5-6/99, S. 26)

\section{Der Autor}

Dr. Wolfram Domke ist wissenschaftlicher Leiter des Steinweg Institutes für psychologische Wirkungsforschung und Projektentwicklung sowie Ausbildungsleiter an der Kölner Akademie für Markt- und Medienpsychologie.

Kontakt: Steinweg Institut, Steinweg 3, 50667 Köln, Tel. 0221/ 2570707 
(c) 20I0 Authors; licensee IÖW and oekom verlag. This is an article distributed under the terms of the Creative Commons Attribution Non-Commercial No Derivates License (http://creativecommons.org/licenses/by-nc-nd/3.o/), which permits unrestricted use, distribution, and reproduction in any medium, provided the original work is properly cited. 